\title{
Modified Z-palatoplasty for correction of acquired nasopharyngeal stenosis following palatal surgery: our experience in nine patients
}

\author{
Mohamed Eesa ${ }^{1}$, Ehsan Hendawy ${ }^{1}$, and Mohammad Waheed El-Anwar ${ }^{1}$ \\ ${ }^{1}$ Zagazig University Faculty of Human Medicine
}

July 13, 2020

\begin{abstract}
Abstract Purpose: to evaluate the safety and the efficacy of a modified Z-palatoplasty (ZPP) technique in management of post-surgical nasopharyngeal stenosis (NPS), describing its steps and results. Methods: This prospective study was conducted on patients with snoring \pm OSA due to acquired post-surgical NPS of grade I and II. Surgical repair using a modified ZPP was employed on the patients and the pre and postoperative results were statistically compared and adverse events were recorded. Results: The grade of NPS improved significantly postoperatively $(\mathrm{p}=0.00136)$ throughout a follow up of one year. Postoperatively, there was statistically significant improvement of AHI $(\mathrm{p}=0.0005)$, VAS of nasal obstruction $(\mathrm{p}<0.0001)$ and VAS of snoring $(\mathrm{p}<0.0001)$. While transient VPI and dysphagia disappeared within 3 months postoperatively. Conclusion: The utilized procedure appears fast, low cost, and easily applicable, and it does not require implants, special tools or suture materials. Furthermore, it gives promising results, with tolerable pain, and rapid recovery without significant or persistent complications.
\end{abstract}

Title

Modified Z-palatoplasty for correction of acquired nasopharyngeal stenosis following palatal surgery: our experience in nine patients.

\section{Succinct key points}

- Nasopharyngeal stenosis (NPS) is one of the challenging problems that can complicate palatal surgeries. Numerous diverse surgical interventions have been used to correct NPS; but, most are complicated and result in inconsistent outcomes

- The aim of the current study wasto evaluate the safety and the efficacy of a modified Z-palatoplasty (ZPP) techniquein the management of post-surgical nasopharyngeal stenosis (NPS), describing its steps and results.

- Surgical repair using a modified ZPP was employed on patients with snoring \pm OSA due to acquired postsurgical NPS of grade I and IIthen the pre and postoperative results were statistically compared and adverse events were recorded.

- The grade of NPS improved significantly postoperatively $(\mathrm{p}=0.00136)$ throughout a follow up of one yearwithsignificant improvement of AHI $(\mathrm{p}=0.0005)$, VAS of nasal obstruction $(\mathrm{p}<0.0001)$ and VAS of snoring $(\mathrm{p}<0.0001)$. While transient VPI and dysphagia disappeared within 3 months postoperatively.

- The utilized procedure appears fast, low cost, and easily applicable, and it does not require implants, special tools or suture materials. Furthermore, it gives promising results, with tolerable pain, and rapid recovery without significant or persistent complications.

Key words: Nasopharyngeal stenosis, palatal surgery, obstructive sleep apnea, snoring, apnea hypoapnea index, UPPP, velopharynx, ZPP. 


\section{Introduction}

Nasopharyngeal stenosis (NPS) is a rare condition of obstruction of the communication between the nasopharynx and oropharynx due to concentric scar contracture of the tonsillar pillars, soft palate, and posterior pharyngeal wall. Mostof NPS nowadays are secondary to adenotonsillectomy, uvulopalatopharyngoplasty (UPPP), or radiotherapy for nasopharyngeal carcinoma ${ }^{1}$. Symptoms of NPS vary from nasal obstruction, difficulty in nose blowing to snoring, hyponasal speech, obstructive sleep apnea (OSA), daytime fatigue, anosmia, rhinorrhea, and dysphagia ${ }^{2}$. With increasing use of electrocautery and different palatal surgeries techniques,NPS incidence also increases ${ }^{3}$.Definitive treatment is often very difficult and may induce more scarring and restenosis. Nonetheless, not much has been written about this challenging complication and unique and standardized management has not yet been presented. Some articles discussed and presented diagrams of flaps that are difficult to understand and would appear to be difficult to reproduce and create, while others presented the idea of stenting ${ }^{4}$ that would appear very difficult to fit for a long time into this irregular, very mobile area. Z-palatoplasty $(\mathrm{ZPP})^{5}$ was first described in 2001 for snoring and OSA but their Z-plasty requires intact anterior and posterior pillars and is meant for patients with intact tonsils. In 2004, Friedman et al described a modified ZPP especially in tonsillectomized patients ${ }^{6}$. Both techniques were dedicated for primary treatment of snoring and OSA. Now, the ZPP role has been diminished due to emergence of many lateral wall adressing techniques. We thought to use a modified form of ZPP as a salvage treatment for post-surgical NPS mainly after UPPP. Our aim was to evaluate the efficacy and safety of a new modified ZPP, describing its steps and results.

\section{Materials and methods}

This prospective study was conducted from April 2016 until December 2019 on patientswith snoring \pm OSA due to post-surgical NPS of grade I and II.

NPS was classified into; grade I: minimal scar tissue, no soft palate lengthening, just adherence between lateral aspects of the palate and posterior pharyngeal wall, grade II: moderate scar tissue, soft palate lengthening, with a small opening in the soft palate $(1-2 \mathrm{~cm}$ in diameter) and grade III: excessive scarring with complete fusion of soft palate and tonsillar pillars with the posterior pharyngeal wall (atresia) or stenosis leaving a pinpoint hole less than $1 \mathrm{~cm}$ in diameter ${ }^{3}$.

Patients with grade III NPS, severe craniofacial anomalies affecting airway, limited mouth opening (interincisive distance $<1.5 \mathrm{~cm}$ ), and/or unfit for general anaesthesiawere excluded.

This study was conducted according to the declaration of Helsinki on Biomedical Research Involving Human Subjects. An informative consent was signed by all patients. The [Blinded for review] University institutional review board approval was obtained.

\section{Outcome measures:}

1. A subjective measure of NPS by upper airways endoscopy and oral cavity exploration.

2. Baseline, 4weeks, 3months, and 1-year Visual Analog Scale (VAS 0-10) for nasal breathing, snoring by bed partner,and dysphagia (0 "no complaints" to 10 "severe complaint").

3. Baseline and 1-year follow-up polysomnography.

4. Short term morbidity and adverse events especially VPI.

Surgical technique (figure 1,2)Under general anaesthesia and patient'ssupine position, mouth gag was inserted. The operative site was infiltrated with $1 \%$ lidocaine with 1:100,000 epinephrine solution.A butterfly mucosal flaps were designed, marked, elevated and removed from the oral surface of the soft palate with caution to preserve the soft palate nasal mucosa. Then, the soft palate was divided in the midline paying attention not to injure the posterior pharyngeal wall mucosa. Excessive soft palate scar tissue was removed as required to facilitate eversion and suturing of the nasal mucosal surface to the oral side. Vicryl suture was placed on the edge of each side of the divided palate to facilitate its eversion. The closure was done in a centrifugal direction by vicryl in 2 layers; $1^{\text {st }}$ one is submucosal inverted sutures and $2^{\text {nd }}$ one is horizontal mattress mucosal sutures without tension and keeping the stitches on the oral side, not on theedge.By 
this way, we aim to change the scar contracture tension lines to anterolateral vectors and to widen the anteroposterior and lateral oropharyngeal air spaces at palatal level. All patients received postoperative paracetamol, antibiotics and steroids, and asked to maintain on soft diet and fluids for 2 weeks. TheSPSS program version 20 (Chicago, Illinois, USA) was used for statistical analysis. P-value $<0.05$ was considered significant.

\section{Results}

Eleven patients were included; nine completed the follow-up; 7 males and 2 females. Their mean age was $47.3 \pm 5.944$ years (range=39-56). Eight patients were caused by UPPP while one caused by adenotonsillectomy. The duration between the causative operation and the NPS repair ranged between 6 and 21 years $($ mean $=1.56 \pm 4.6)$. Preoperatively, the NPS was grade 1 in two patients $(22.2 \%)$ and grade 2 in 7 patients $(77.8 \%)$.

The operative bleeding was non-significant and recovery was eventless in all cases. The mean postoperative hospitalization was $1.8 \pm 1.03$ (range=1-4) days. Noinfection, primary or secondary hemorrhages were reported. Postoperative pain was tolerated and controlled by paracetamol orally and was completely relieved within 2 weeks. All patients resumed normal diet after 2 weeks. Five patients developed transient VPI that was noted occasionally with drinking fluids, but did not interfere with normal diet and disappeared completely within 3 months with no permanent VPI. No patients developed palatal fistula or restenosis $>50 \%$.

The grade of NPS improved significantly postoperatively $\left(\mathrm{X}^{2}=13.2, \mathrm{p}=0.00136\right)$ throughout the follow up of one year. Preoperatively, all patients had OSA that was objectively documented by polysomnography

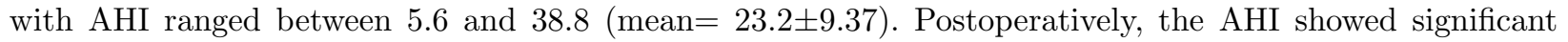
improvement to a mean of $12.35 \pm 3.75(\mathrm{t}=5.0089, \mathrm{p}=0.0005)$ (Table 1$)$.

The encountered complications were temporary and related to postoperative dry throat, and inability to clear the throat. Dysphagia showed early worsening but it improved completely at three months postoperatively and remained throughout the follow-up period (Table 2).

\section{Discussion}

NPS is a challenging problem that can complicate palatal surgeries. Diversesurgical interventions have been used to correct NPS; but, most are complicated and result in inconsistent outcomes ${ }^{4}$.

Theend-goal of repair remains the expansion of the nasopharyngeal-oropharyngeal communication through removal/release of the scar and mucosal coverage of denuded surfaces to limit recurrence. MacKenty described the first use of superiorly based pharyngeal mucosal flaps folded on themselves in $1927^{2}$. This was subsequently modified by Kazanjian et $\mathrm{al}^{7}$ to include local oropharyngeal mucosal flaps. In more severe NPS cases, aggressive strategies included use of radial forearm and jejunal free flaps to treat recurrent stenosis after conventional flaps failure. Due to technical challenges of free tissue transfer, other local approaches developed; bilateral Z-plasty ${ }^{8}$ andbivalved palatal transposition flap ${ }^{9}$.

The NPS repair is difficult because it is rare clinical entity. Clinical series are usually limited to just a few patients and comparative studies are non-existent. Few reports in the literature described the usage of anyone specific technique for its surgical correction with lacking follow-up beyond 6 months in most series. In addition, there are no standardized outcome measures yet to detect the effectiveness of each surgical method. The success has been based mostly on subjective relief of symptoms ${ }^{9}$. But, we used the subjective VAS of symptoms, grading of stenosis and objective evaluation by AHI.

Most acquired NPS arise after UPPP. In the current study, UPPP was the cause in $88.9 \%$ of cases and males were more affected than females. Similarly, the two studied cases by Toh et $\mathrm{al}^{9}$ and the three repaired by Magdy et $\mathrm{al}^{4}$ were males.

Successful NPS correction relies on adequate scar tissue removal and/or lysis, and coverage of the raw mucosal surfaces $^{9}$. So, we ensured complete scar tissue removal with mucosa preservation to use it to cover the raw 
area and avoid the presence of two raw areas on the nasopharynx and palate.

In our study, there was no necessity for postoperative obturationthat appears very difficult to fit for a long time into this irregular, very mobile and sensitive mucosal area and may be difficult to be tolerated by the patients beside its foreign body effect and reaction.

The current procedure was performed through the transoral rout without extra tool or suture material and so no financial implications.

Avoidanceof excess palatal dissection reduced postoperative edema and soavoided ICU admission. Our followup period (12 months at least) exceeds these of past studies and thus the results appear promising.

Recently, Cammaroto et $\mathrm{al}^{10}$ published a technique for grade III NPS correction that represents a modification of bivalved palatal transposition flaps originally described by Toh et $\mathrm{al}^{9}$. However, our technique which represents a modified form of ZPP originally described by Friedman et al dedicated mainly for grade I and II NPS.

Thus, our technique for NPS repair showed satisfactory outcome and easy applicability. However, further studies on larger number of patients and in comparison to other techniques are recommended.

\section{Conclusion}

The described procedure appears to be effective, fast, low cost, and easily applicable, and it does not require implants, special tools, or sutures materials without significant complications.

\section{Conflict of interest}

The authors declare no conflict of interest.

\section{References}

1- Giannoni C, Sulek M, Friedman EM, Duncan NO III. Acquired nasopharyngeal stenosis: a warning and review.Arch Otolaryngol Head Neck Surg 1998; 124: 163-167.

2- Mackenty J. Nasopharyngeal atresia, Arch. Otolaryngol . 1927; 6: 1-27.

3- Krespi YP, Kacker A. Management of nasopharyngeal stenosis following uvulopalatoplasty, Operative Techniques in Otolaryngology-Head and Neck Surgery 2002; 13(2): 161-165.

4- Madgy DN, Walter Belenky W, Dunkley B, Shinhar S. A simple surgical technique using the plasma hook for correcting acquired nasopharyngeal stenosis. Laryngoscope 2005; 115: 370- 372.

5- Viera MBM, Leite SHP, Cunha FC. (2001) Zetapalatopharyngoplasty (ZPPP) new surgical technique for snoring and sleep apnea treatment.Braz J Otolaryngol 67:1-8.

6- Friedman M, Ibrahim HZ, Vidyasagar R, Pomeranz J, Joseph NJ. (2004) Z-palatoplasty (ZPP) a technique for patients without tonsils. Otolaryngol Head Neck Surg. 131(1):89-100. doi:10.1016/j.otohns.2004.02.051

7- Kazanjian VH, Holmes EM. Stenosis of the nasopharynx and its correction.Arch Otolaryngol .1946; 44: 261-273.

8- Wan DC, Kumar A, Head CS, Katchikian H, Bradley JP. Amelioration of acquired nasopharyngeal stenosis, with bilateral Z-pharyngoplasty, Ann.Plast. Surg . 2010; 64 (6): 747-750.

9- Toh E, Pearl AW, Genden EM, Lawson W, Urken ML, Bivalved palatal transposition flaps for the correction of acquired nasopharyngeal stenosis. American Journal of Rhinology 2000; 14: 199-204.

10- Cammaroto G, Stringa LM, Cerritelli L, Bianchi G, Meccariello G, Gobbi R, Iannella G, Magliulo G, Zhang H, Baghat AY, Galletti F, Pelucchi S, Stomeo F, Younes MB, AlAjmi M, De Vito A, Vicini C. Acquired nasopharyngeal stenosis correction using a modified palatal flaps technique in obstructive sleep apnea (OSA) patients. Int. J. Environ. Res. Public Health 2020; 17: 2048. 


\section{Figure legends}

Figure 1: A; Mucosal flaps designed and marked. B;Elevating and removing mucosa from the oral surface of soft palate.C; Dividing soft palate in midline. D; Eversion and suturing the nasal mucosa to the oral one.

Figure 2: Pre and post-operative view of the palate.

Tables

Table 1 .Preopeative and 1-year postoperative follow up.

\begin{tabular}{llllll}
\hline $\begin{array}{l}\text { Outcome } \\
\text { measures }\end{array}$ & $\begin{array}{l}\text { Outcome } \\
\text { measures }\end{array}$ & Preop & $\begin{array}{l}\text { 1 year } \\
\text { postop }\end{array}$ & Test & P value \\
\hline $\begin{array}{l}\text { Grade of } \\
\text { stenosis }\end{array}$ & Grade $\mathbf{0}$ & 0 & 6 & $\mathrm{X}^{2}=13.2$ & $0.00136 \mathrm{~S}$ \\
& Grade 1 & 2 & 3 & & \\
& Grade 2 & 7 & 0 & & \\
AHI & AHI & $27.3 \pm 6.276$ & $12.35 \pm 3.75$ & $\mathrm{t}=5.0089$ & $0.0005 \mathrm{~S}$ \\
Nasal & Nasal & $6.78 \pm 0.916$ & $0.3 \pm 0.67$ & $\mathrm{t}=17.1295$ & $<0.0001 \mathrm{~S}$ \\
breathing & breathing & & & & \\
Snoring & Snoring & $6.25 \pm 0.968$ & $0.67 \pm 0.94$ & $\mathrm{t}=12.4064$ & $<0.0001 \mathrm{~S}$ \\
Dysphagia & Dysphagia & $3.5 \pm 0.866$ & 0 & $\mathrm{t}=12.1247$ & $<0.0001 \mathrm{~S}$ \\
\hline
\end{tabular}

Legends: AHI, apnea hypoapnea index; S, significant

Table 2 . Preoperative and postoperative VAS of symptoms

\begin{tabular}{lllllll}
\hline VAS & & Preop VAS & One month postop & 3 months post & 1 year post & Statistical test \\
\hline Nasal breathing & Range & $5-8$ & $1-4$ & $0-2$ & $0-1$ & \\
& Mean & $6.78 \pm 0.916$ & $2.44 \pm 0.83$ & $0.3 \pm 0.67$ & $0.2 \pm 0.4$ & $\mathrm{~F}=159.669$ \\
Snoring & Range & $5-8$ & $1-5$ & $0-2$ & $0-2$ & \\
& Mean & $6.25 \pm 0.968$ & $3 \pm 1.3$ & $0.67 \pm 0.94$ & $0.67 \pm 0.94$ & $\mathrm{~F}=58.112$ \\
Dysphagia & Range & $2-5$ & $3-6$ & 0 & 0 & \\
& Mean & $3.5 \pm 0.866$ & $4.2 \pm 0.916$ & 0 & 0 & $\mathrm{~F}=113.788$ \\
\hline
\end{tabular}

Legend: $F=A N O V A$ test; $S=$ significant

\section{Hosted file}

Table 1.docx available at https://authorea.com/users/341855/articles/468760-modifiedz-palatoplasty-for-correction-of-acquired-nasopharyngeal-stenosis-following-palatalsurgery-our-experience-in-nine-patients

\section{Hosted file}

Table 2.docx available at https://authorea.com/users/341855/articles/468760-modifiedz-palatoplasty-for-correction-of-acquired-nasopharyngeal-stenosis-following-palatalsurgery-our-experience-in-nine-patients 

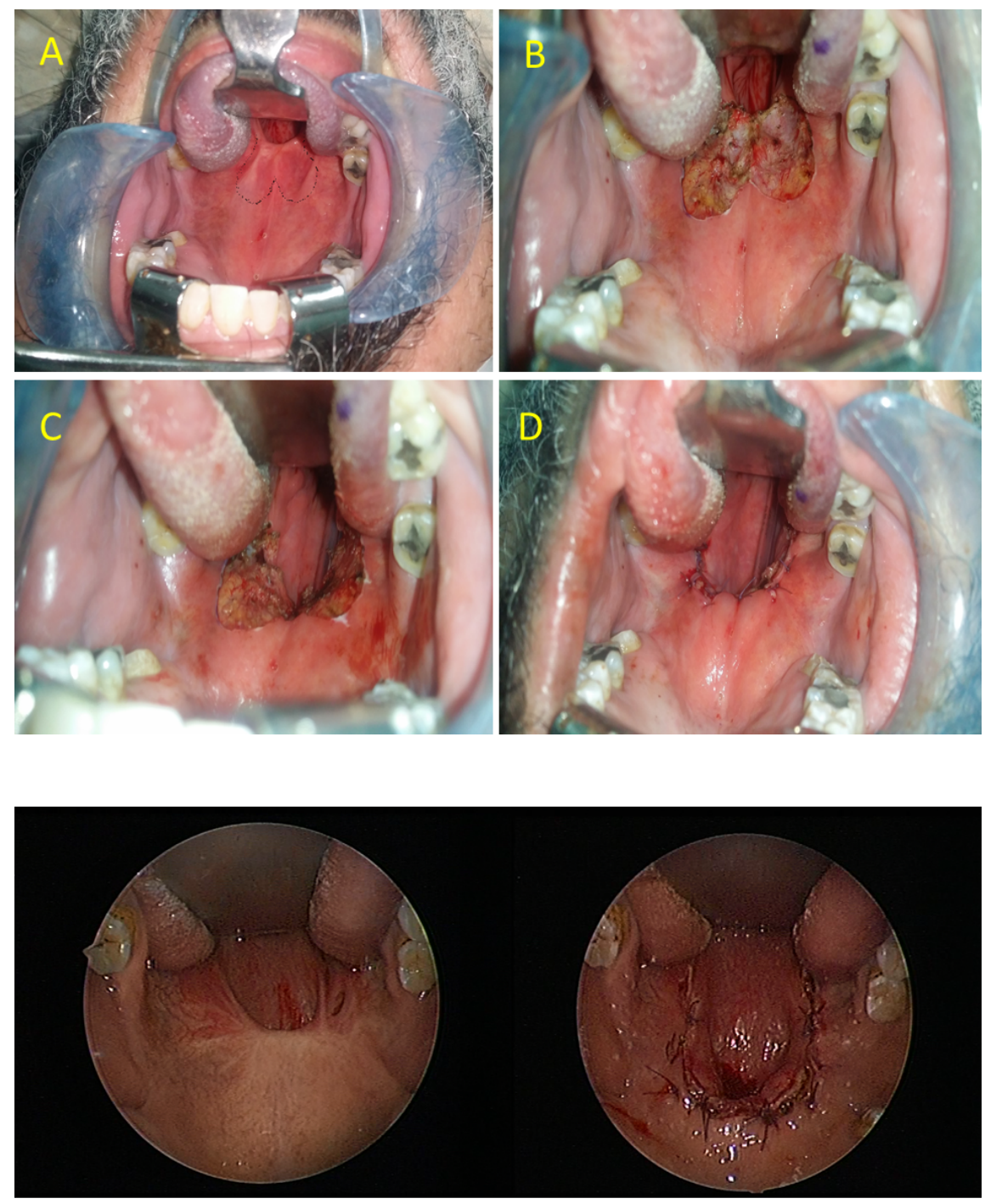

PRE

POST 